\title{
Fatal Atlanto-Axial Luxation in Rheumatoid Arthritis
}

\author{
By Wulliam Martel and Murray R. Abell
}

Fatal atlanto-axial dislocation occurred in two patients with rheumatoid arthritis and in both there were severe degenerative changes in the spinal ligaments and skeletal muscle in this area. Attention is called to the frequency of spontaneous cervical dislocations in rheumatoid patients and the advisability of obtaining routine radiographic examination of the cervical spine in patients with advanced disease.
Mortal dislocation atlanto-axial occurreva in duo patientes con arthritis rheumatoidee. Ambes monstrava sever alterationes degeneratori in le ligamentos spinal e le musculo skeletic in iste area. Es signalate le frequentia de spontanee dislocationes cervical in patientes rheumatoidee e le desirabilitate de obtener routinarimente examines radiographic del spina cervical in patientes con morbo avantiate.

$\mathbf{T}$

HE CERVICAL SPINE is frequently affected in rheumatoid arthritis, and subluxations are common, particularly of the atlanto-axial joints. Neurologic manifestations often are absent, even in patients with severe atlanto-odontoid separation.1,2 However, symptoms of compression of the spinal cord and medulla may be observed and, unless the possibility of cervical luxation is considered, the correct diagnosis may not be appreciated, particularly in patients with crippling joint disease in whom neurologic examination is difficult. This report of two patients who sustained atlanto-axial luxations which were diagnosed only at necropsy emphasizes the potential danger of this complication.

\section{Report of Cases}

\section{Case 1 (M. M. \#416698)}

This white female was first seen in 1938 at the age of 46 years with arthritis of her peripheral joints for 5 years. Physical examination showed active synovitis of her hands, wrists, feet, elbows, ankles and knees. Subcutaneous rheumatoid nodules were noted. No symptoms were referable to the spine but moderate tenderness was elicited, over the spinous process of C7. Roentgenograms of the hands and wrists were characteristic of rheumatoid arthritis. The patient was treated with aspirin for several years during which time the disease showed moderate progression. In 1941 and 1942 some of the subcutaneous nodules were removed and the rheumatoid nature confirmed. She was under the care of a private physician between 1943 and 1953 and, to our knowledge, she had not received steroid therapy.

The patient's final admission to The University of Michigan Medical Center was on March 23, 1953, because of nausea, vomiting, and photophobia. She had had a non-productive cough, progressive weakness, anorexia, and weight loss for several weeks. Her husband related that 10 days prior to admission the patient became "extremely anxious" but was unable to explain the basis for her fear. On several occasions during this period she expressed paranoid ideas and was disoriented. There were no localizing neurologic signs. Physical examination showed an emaciated, chronically ill, white female with severe deformities of peripheral joints and subcutaneous nodules. She was afebrile, and respirations,

From the Departments of Radiology and Pathology, The University of Michigan, Ann Arbor, Mich. 
blood pressure, and pulse were normal. She had extreme photophobia and a dilated, fixed left pupil.

Neck motion was limited and she was unable to raise her head. Cardiac examination was negative, and auscultation of the lungs revealed a few moist rales posteriorly at the bases. The hemoglobin level was $11 \mathrm{Gm}$. per cent; leukocyte count was 5300/cu.mm. with a normal differential count, and the erythrocyte sedimentation rate (Wintrobe, corrected) was $32 \mathrm{~mm}$. $/ \mathrm{hr}$. A serologic test for the rheumatoid factor was not done. She had periods of complete disorientation, with vomiting, and a diagnosis of acute primary glaucoma was made. Tension in the left eye was $72 \mathrm{~mm}$. A left iridectomy was performed under local anesthesia on March 27, 1953, and several hours later she developed grand mal seizures, generalized flaccidity with hyperactive deep tendon reflexes on the left, and bilateral positive Babinski signs. She did not regain consciousness and expired on April 1, 1953. The clinical impression was that the patient had suffered a cerebral vascular accident due to atherosclerosis.

Necrospy findings: The necropsy was performed 10 hours after death. Post-mortem culture of blood from the heart yielded no growth. The body length was 64 inches and the weight $110 \mathrm{lb}$.; general nutrition was poor. Superficial decubiti were present over the sacrum and lower extremities. There was marked ulnar deviation of the hands, deformities and subluxations of several joints of the hands and feet, and subcutaneous nodules. After removal of the brain, examination of the base of the skull disclosed a nodular projection beneath the dura in the left side of the foramen magnum. This represented the odontoid process which compressed and forced the spinal cord and medulla oblongata to the right. The altered position of the odontoid process was due to an atlanto-axial dislocation with excessive mobility of the atlas; there was no fracture and no abnormality of the other vertebrae. There was congestion, edema, and some hemorrhage in the covering meninges and the adjacent soft tissues of the neck. The brain weighed $1280 \mathrm{Gm}$. There was minimal atherosclerosis of the vertebral and cerebral arteries with no points of stenosis and no thrombi. Thin sections of brain disclosed no infarcts, hemorrhage, or neoplasm. The main thoraco-abdominal incision revealed a small amount of peritoneal and bilateral pleural transudate. The heart weighed $250 \mathrm{Gm}$. and the only change was a moderate degree of brown atrophy and calcification of widely patent coronary arteries. The lungs weighed $390 \mathrm{Gm}$. and $420 \mathrm{Gm}$. and contained several fibronecrotic nodules up to $1 \mathrm{~cm}$. in diameter. The thyroid gland was atrophic, weighing only $5 \mathrm{Gm}$. The spleen, pancreas, liver, kidneys, and adrenal glands were grossly normal. There was an acute superficial ulcer $1.5 \mathrm{~cm}$. in diameter on the posterior wall of the pylorus, and diverticula were present in the colon. The ovaries were atrophic.

Microscopic findings: Sections of the proximal portion of the spinal cord and the adjacent medulla oblongata revealed a moderate degree of acute congestion with scattered recent hemorrhages, some fragmentation, and edema. There was no significant inflammatory cell infiltrate or demyelinization and only questionable recent necrosis. There was some cerebral edema and minimal focal gliosis of the brain, but no necrosis, hemorrhage, or neoplasm. The ligaments of the upper vertebrae, including the transverse ligament, had areas of degeneration and increased vascularity; no rheumatoid nodules were present. These tissues lacked the normal homogeneous compactness and varied in staining density, with the degenerative areas appearing granular and reticulated. The attached connective tissues were increased in vascularity and an occasional small artery was involved in and surrounded by inflammatory cells indicative of a vasculitis (fig. 1). There was advanced degeneration, atrophy and focal necrosis in the paravertebral muscles. The fibers varied in size and staining reaction and were granular and vacuolated. The sarcolemmal nuclei were focally prominent and a few inflammatory cells were related to the scattered necrotic muscle fibers (fig. 2). There was minimal vasculitis within the muscle. Similar changes were found in muscles and vessels in other areas of the body but were not as prominent.

Other histologic findings included subcutaneous rheumatoid nodules (fig. 3), patchy, non-specific pulmonary fibrosis, old calcified pulmonary and hepatic granulomas, erosive esophagitis, and atrophy of the thyroid gland. 


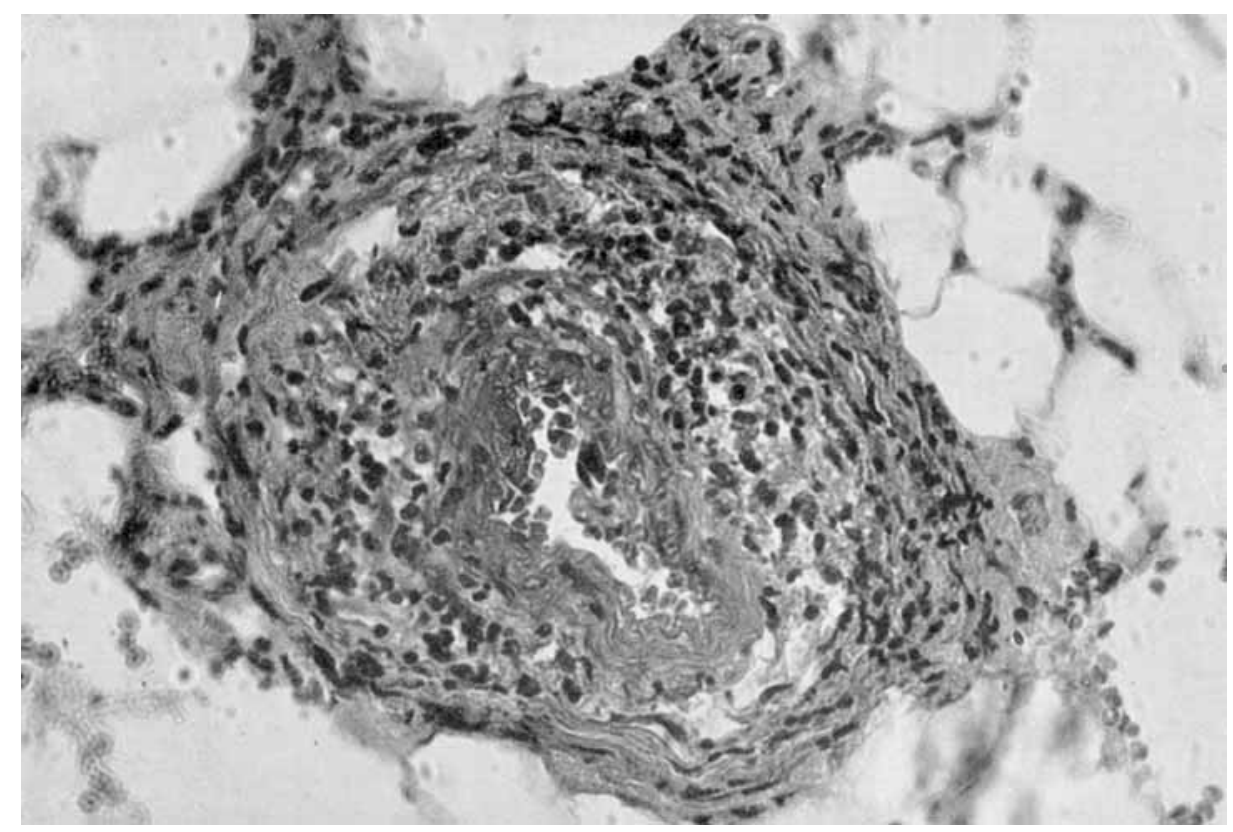

Fig. 1.-Case 1. A small artery in periosseous fibroadipose tissue of neck. There is necrosis, edema, and inflammatory cell infiltration of media. H. \& E. X330.

The final anatomical diagnosis was chronic rheumatoid disease with arthritis, subcutaneous nodules, myopathy, and vasculitis. Death was attributed to anterior luxation of the atlas on the axis with compression of the spinal cord and medulla oblongata.

\section{Case 2 (J. S. \#593317)}

A 67 year old white male had had severe, widespread rheumatoid arthritis for 14 years and was admitted to the University Hospital on June 7, 1960, with persistent lethargy and disorientation which had developed suddenly 3 days prior to admission. He had had a painful swelling over the left ankle for 5 days, with fever and malaise, and several small "abscesses" on the sole of the right foot had recently been incised and drained. He had had a "stroke" 5 months earlier without neurologic sequelae. The arthritis had progressed in the last 2 years so that the patient was unable to stand without support and he had developed multiple decubiti. He had not received steroid therapy.

Physical examination revealed a chronically ill male. Body temperature was $103 \mathrm{~F}$., pulse rate 104/min., and blood pressure 100/60 mm. Hg. He had bilateral cataracts (unspecified type) and the neck was supple. There was dullness to percussion at the base of the right lung with diminished breath sounds and rales. He had severe arthritis of the hands and wrists with advanced deformities and subluxations. The ankles, knees, hips, elbows, and shoulders also were involved. The left ankle was particularly swollen, hot, and tender, due to lymphangitis. A draining ulcer was seen over the acromion process of the right scapula, and subcutaneous rheumatoid nodules were noted in the forearms. Peripheral pulses were normal and, other than aphasia, the neurologic examination was negative. The hemoglobin level was $11.5 \mathrm{Gm}$. per cent. The erythrocyte sedimentation rate was $12 \mathrm{~mm}$./hr. and the leukocyte count was $4000 / \mathrm{cu} . \mathrm{mm}$. with the following differential: 60 per cent polymorphonuclear cells; 27 per cent lymphocytes; 10 per cent monocytes, and 1 per cent basophils. The serum uric acid and blood urea nitrogen levels were normal. The latex fixation test for the rheumatoid factor was positive (1:5120). Roentgenograms of the hands and ankles were characteristic of rheumatoid arthritis; films of the cervical spine 


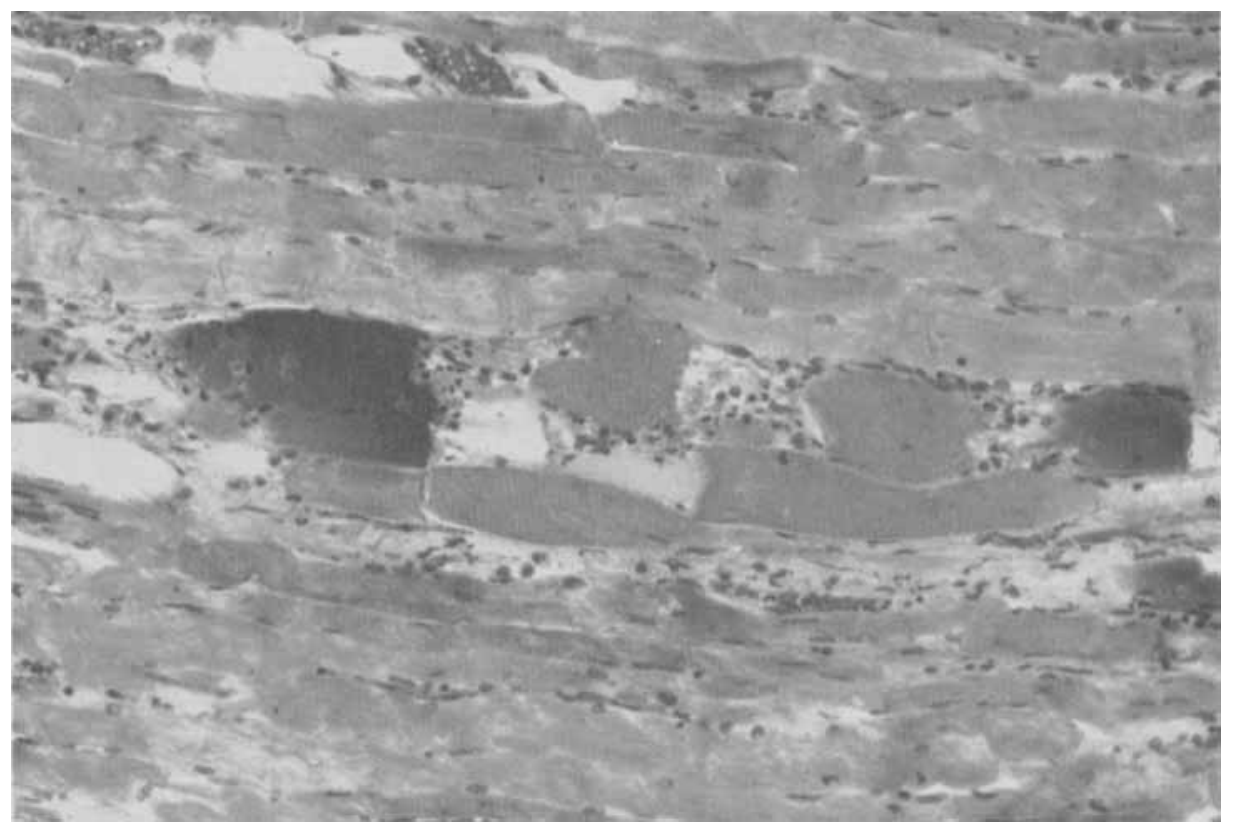

Fig. 2.-Case 1. Advanced degeneration and focal necrosis in paravertebral muscle. The irregularly swollen and deeply stained fibers in the center of the field are necrotic; they are partially surrounded by and infiltrated by neutrophils. H. \& E. X180.

were not obtained. A chest film was interpreted as possible pneumonia at the base of the right lung. Purulent material was aspirated from the soft tissues adjacent to the left ankle and a blood culture revealed hemolytic, coagulase positive Staphylococci. The patient showed considerable improvement while receiving penicillin and his sensorium cleared, but respirations ceased suddenly on the 5 th day of hospitalization. His death was attributed clinically to a cerebral vascular accident.

Necropsy findings: The necropsy was performed 8 hours after death. Culture of blood from the heart yielded no growth. General nutrition was very poor with extensive muscular wasting and decubiti; the body weight was $91 \mathrm{lb}$. and the length 66 inches. Marked rheumatoid deformities of the hands, wrists, ankles, knees, and feet were noted, as well. as subcutaneous nodules and residual cellulitis over the right ankle.

The base of the skull, after removal of the brain, disclosed the odontoid process projecting into the foramen magnum toward the right and occupying about one fourth of the foramen. The process compressed the anterior aspect of the medulla oblongata and it could be replaced by traction and extension of the head. The supporting ligaments, including the transverse ligament, were lax and resulted in extreme mobility of the atlanto-odontoid joints. The remaining vertebrae were osteoporotic but otherwise normal. The brain weighed $1400 \mathrm{Gm}$. A few atheromatous plaques were scattered throughout the basal arteries but did not significantly compromise the lumens and no thrombi were found. Transections of the brain disclosed no hemorrhages or infarcts; the only suggested alteration was some softening in the region of the inferior olivary nuclei. The heart weighed $260 \mathrm{Gm}$. A moderate degree of coronary arterial atherosclerosis was noted and the vessels were widely patent. The lungs weighed $340 \mathrm{Gm}$. and $440 \mathrm{Gm}$. and revealed focal emphysema and minimal, patchy, lobular pneumonia. The spleen was enlarged, soft, and weighed $470 \mathrm{Gm}$. Other organs were normal.

Microscopic findings: The proximal end of the spinal cord and medulla oblongata were 


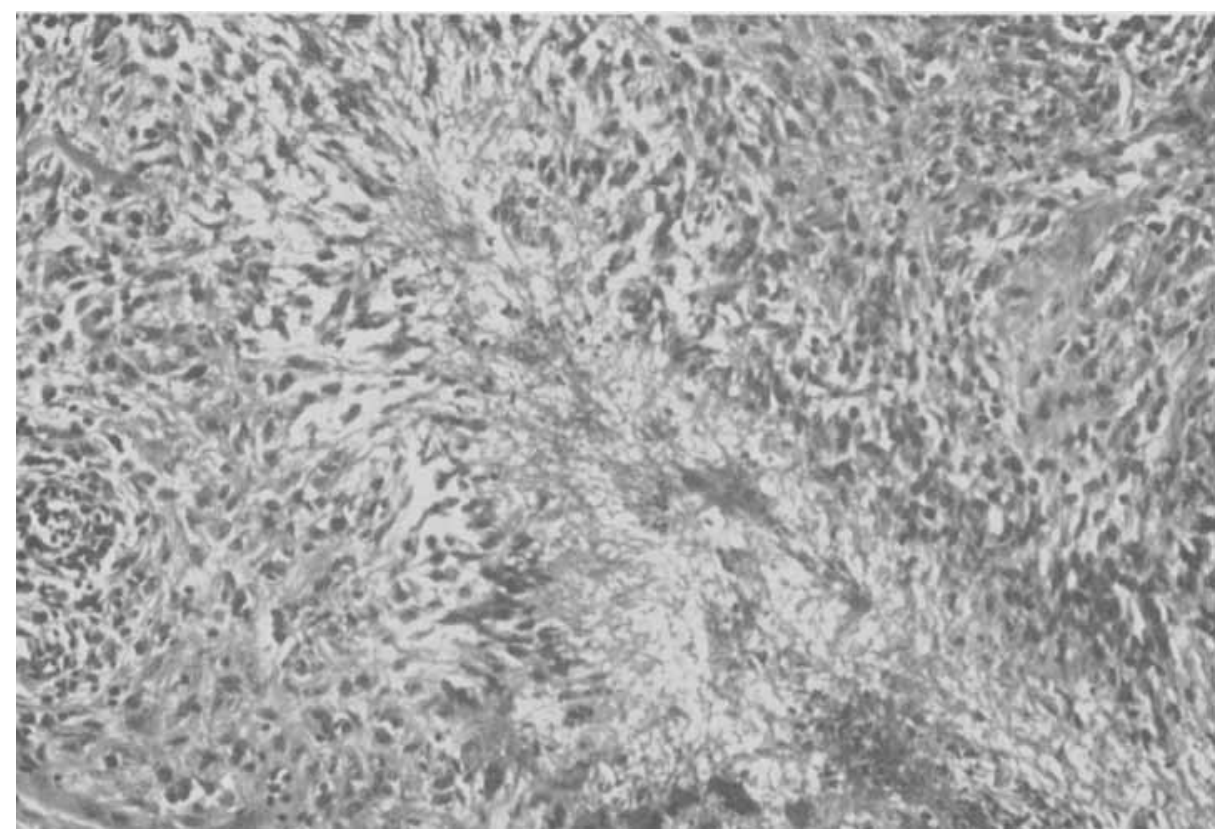

Fig. 3.-Case 1. A subcutaneous rheumatoid nodule. There is a central area of fibrinoid necrosis surrounded by spindled mesenchymal cells and fibrous tissue with focal collections of chronic inflammatory cells. H. \& E. X170.

congested and edematous and contained small recent hemorrhages; the adjacent meninges also were congested. The ligaments, including the transverse ligament, in the region of the upper vertebrae were edematous and showed degenerative changes. The synovial cells were hyperplastic and there were occasional subsynovial collections of lymphocytic cells. At one level there was some fibrin deposited on the synovial surface. The changes, although definite, were relatively minimal and non-specific. Scattered collections of lymphocytes were present in the perivertebral muscles (fig. 4). There was focal atrophy of muscle fibers, no active vasculitis, but the walls, particularly the intima, of some arterioles were thickened and fibrotic.

Other findings included pneumonitis, interstitial pulmonary fibrosis, focal emphysema, and several anthracofibrotic pulmonary nodules which probably represented old anthracosilicosis. There was a moderate degree of coronary arterial atherosclerosis without myocardial change. A well-differentiated adenocarcinoma was found in the prostate.

The final pathologic diagnosis was rheumatoid disease with death attributed to atlantoaxial luxation and compression of the spinal cord and medulla. Chronic pulmonary disease and adenocarcinoma of the prostate gland did not appear to have contributed significantly to the death.

\section{Discussion}

Sharp and Purser ${ }^{2}$ recently described the clinical manifestations of atlantoaxial dislocation in 26 patients with rheumatoid arthritis. They noted that characteristic pain in the upper cervical or occipital region was the presenting symptom in eight patients and neurologic manifestations were the initial findings in five others. In the remaining 13 patients the displacement was not suspected until radiographs of the cervical spine were examined, and in none could a definite diagnosis of atlanto-axial dislocation be made clinically. 


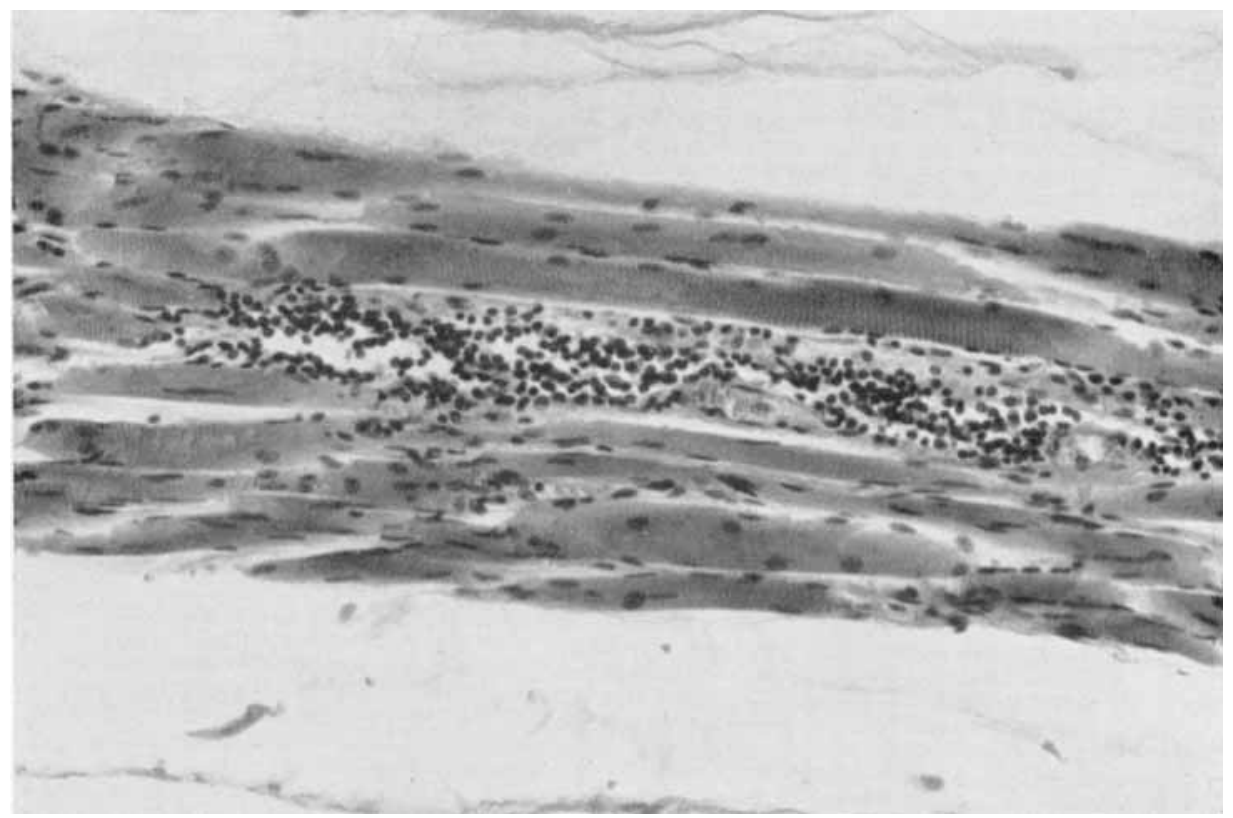

Fig. 4.-Case 2. There is atrophy of this skeletal muscle and a focal infiltrate of lymphocytes. H. \& E. X170.

Further inquiry failed to elicit a history of suboccipital pain in six of the patients. It is significant that cervical spine films were not obtained in either of our two cases. There have been instances in which such atlanto-axial dislocation was the basis for sudden, fatal compression of the spinal cord and medulla. Storey ${ }^{3}$ described a patient who had rheumatoid arthritis for only 4 years and in whom severe spinal cord compression was verified at necropsy. Davis and Markley ${ }^{4}$ reported a patient who developed striking and progressive bulbar symptoms, and necropsy disclosed destruction of the atlas and base of the skull with herniation of the odontoid through the foramen magnum, and compression of the medulla. The problem takes on added significance when it is realized how commonly atlanto-axial dislocation occurs in patients with rheumatoid arthritis. Ball ${ }^{5}$ noted that eight of 20 patients with severe rheumatoid arthritis at necropsy had atlanto-axial subluxations. Sharp and Purser ${ }^{2}$ found that 18.9 per cent of hospital in-patients with rheumatoid arthritis showed this abnormality and, more recently, routine radiologic examination of 97 consecutive clinic patients disclosed a prevalence of 35 per cent and in these patients the subluxation was severe in 19 per cent. ${ }^{6}$ The importance of diagnosing this condition, even if asymptomatic, cannot be over-emphasized. Treatment with a supportive collar, particularly in severe cases, may prevent neurologic complications; cervical manipulation should be avoided.

The radiologic features of rheumatoid arthritis of the cervical spine have been described and the necessity of including a lateral view in flexion has been stressed. ${ }^{1}$ Frequently, the dislocation can only be appreciated when the head is in the flexed position. Midline lateral laminagraphy may be helpful 
in disclosing erosions of the odontoid process and may occasionally show periosteal new bone extending into the peri-odontoid soft tissues. ${ }^{7}$ However, the uncalcified retro-odontoid soft tissues cannot be evaluated without myelography since the quantity of epidural fat in this vicinity is negligible." Furthermore, the blurred shadows of the lateral masses of $\mathrm{Cl}$ and the mastoid processes often cause confusing shadows in this area on linear laminagrams, even in midline sections.

Neurologic manifestations in these individuals may be due to vertebral artery insufficiency or compression of the spinal cord, medulla, or nerve roots. In older individuals the ischemic effect of cerebral arteriosclerosis may be aggravated. The laxity of ligaments and supportive tissues in the two cases of this report allowed luxation and may be attributed to the changes in ligaments and muscles in the area. Rupture of the transverse ligament need not be present. It is pertinent that neither of these patients received steroid therapy. The patient may have a vague apprehensive sensation that something has developed in addition to the arthritis. An episode of extreme anxiety was implied in Case 1. Sharp and Purser ${ }^{2}$ described a patient (Case 47) who had neurologic manifestations and also developed a vague sensation of dread.

\section{SUMMARY}

Two instances of sudden death in patients with rheumatoid disease due to atlanto-axial dislocation are described. There were severe degenerative changes in ligaments and skeletal muscle in the area which could explain the laxity of ligaments and supportive tissues. Subluxation of these vertebrae is much more common than is generally realized. Routine radiographic examination of the cervical spine, with flexion, should be employed in all patients with advanced rheumatoid disease to detect this complication.

\section{REFERENCES}

1. Martel, W.: The occipito-atlanto-axial joints in rheumatoid arthritis and ankylosing spondylitis. Am. J. Roentgenol. 86:223, 1961.

2. Sharp, J., and Purser, D. W.: Spontaneous atlanto-axial dislocation in ankylosing spondylitis and rheumatoid arthritis. Ann. Rheumat. Dis. 20:47, 1961.

3. Storey, G.: Changes in the cervical spine in rheumatoid arthritis with compression of the cord with reports of four cases. Ann. Phys. Med. 4:216, 1958.
4. Davis, F. W., Jr., and Markley, H. E.: Rheumatoid arthritis with death from medullary compression. Ann. Int. Med. $35: 451,1951$.

5. Ball, J.: Quoted by Sharp, J., and Purser, D. W.2

6. Martel, W., and Duff, I. F.: Unpublished data.

7. -, Holt, J. F., and Cassidy, J. T.: Roentgenologic manifestations of juvenile rheumatoid arthritis. Am. J. Roentgenol. 88:400, 1962.

"Observation in conjunction with work done with Dr. Thomas M. Oelrich, Department of Anatomy, The University of Michigan Medical Center. 
William Martel, M.D., Assistant Professor of Radiology, Department of Radiology, The University of Michigan, Ann Arbor, Mich.

Murray R. Abell, M.D., Ph.D., Professor of Pathology, Department of Pathology, The University of Michigan, Ann Arbor, Mich. 